Research Article

\title{
Growth Inhibition of a Novel Iron Chelator, DpdtC, against Hepatoma Carcinoma Cell Lines Partly Attributed to Ferritinophagy-Mediated Lysosomal ROS Generation
}

\author{
Tengfei Huang, ${ }^{1}$ Yanjie Sun, ${ }^{1,2}$ Yongli Li, ${ }^{3}$ Tingting Wang, ${ }^{1}$ Yun Fu, ${ }^{1,4}$ Cuiping Li, ${ }^{1,4}$ \\ and Changzheng $\mathrm{Li} \mathbb{D}^{1,2,4}$ \\ ${ }^{1}$ Department of Molecular Biology and Biochemistry, Xinxiang Medical University, Xinxiang, Henan 453003, China \\ ${ }^{2}$ Experimental Teaching Center of Biology and Basic Medicine, Sanquan College of Xinxiang Medical University, Xinxiang, \\ Henan 453003, China \\ ${ }^{3}$ Department of Histology and Embryology, Sanquan College of Xinxiang Medical University, Xinxiang, Henan 453003, China \\ ${ }^{4}$ Laboratory of Molecular Medicine, Xinxiang Medical University, Xinxiang, Henan 453003, China
}

Correspondence should be addressed to Changzheng Li; changzhenl@yahoo.com

Received 6 April 2018; Revised 10 June 2018; Accepted 8 July 2018; Published 5 August 2018

Academic Editor: Julia M. dos Santos

Copyright (C) 2018 Tengfei Huang et al. This is an open access article distributed under the Creative Commons Attribution License, which permits unrestricted use, distribution, and reproduction in any medium, provided the original work is properly cited.

\begin{abstract}
Some iron chelators display significant anticancer activity that may involve ferritin degradation either in proteasomes or in lysosomes, and the latter might involve ferritinophagy with a period. However, the correlation of ferritinophagy with anticancer activity of iron chelator was not fully determined. Revealing the underlying link therefore is required. Di-2-pyridylketone dithiocarbamate (DpdtC), a novel iron chelator, could mobilize iron from ferritin and displayed excellent antitumor against hepatoma carcinoma cell lines $\left(\mathrm{IC}_{50 \mathrm{~s}}=0.4 \pm 0.2\right.$ for $\mathrm{HepG} 2$ and $3.5 \pm 0.3 \mu \mathrm{M}$ for Bel-7402, resp.); we speculated that the antiproliferative action of DpdtC might involve ferritinophagy. To this end, the alterations of ferritin, microtubule-associated protein light chain 3 (LC3-II), and nuclear receptor coactivator 4 (NCOA4) were investigated after exposure of DpdtC to the cells. The results revealed that DpdtC could cause increases of autophagic vacuoles and LC3-II. The data from cellular immunofluorescence and Western blotting showed a reciprocal relation between abundances of ferritin and LC3-II, but the trends of NCOA4 and LC3-II in abundance were in a similar manner, indicating that a ferritinophagy occurred. Further studies revealed that the ferritinophagy evoked an iron-driven intralysosomal oxidative reaction, resulting in LMP change and lipid peroxidation. Thus, a ferritinophagy-mediated lysosomal ROS generation playing a role in the antiproliferative action of $\mathrm{DpdtC}$ could be proposed, which will enrich our knowledge of iron chelator in cancer therapy.
\end{abstract}

\section{Introduction}

Iron is an essential element and plays a crucial role in cellular proliferation and DNA synthesis. In biological systems, iron acts as a cofactor in biochemical processes, such as oxygen storage, oxidative phosphorylation, and enzymatic reactions or presents as free ion (labile iron pool (LIP)) that generate reactive oxygen species (ROS) via the Fenton reaction, therefore the level of free iron in a cell must be tightly controlled $[1,2]$. It has been demonstrated that the LIP is regulated by ferritin, a highly conserved iron storage protein, which is composed of two subunits, $\mathrm{H}$-ferritin and L-ferritin, and the twelve pairs of subunits binding head to foot form the 24 -subunit ferritin cage $[3,4]$. When the iron level in the cell is low, ferritin is degraded allowing the release of iron for use by the cell.

In addition, some reductants or iron chelators could extract iron from ferritin, which was considered to be due to the small reductants or chelators entering to the interior of the ferritin molecule through three-fold channels in the protein shell, but not for larger ones [5]. Although iron chelators lead to iron poor ferritins in vitro, there is no evidence that iron can exit ferritin prior to ferritin degradation in cellular level [6]. Iron chelators can induce ferritin 
degradation that occurs either in lysosomes or in proteasomes. Generally, the worn-out proteins or organelle can be degraded in lysosomes, and microtubule-associated protein light chain 3 (LC3) is involved in the proteolytic process; consequently, LC3 is considered as an autophagy indicator. During autophagy, cytosolic form of LC3 (LC3-I) is conjugated to phosphatidylethanolamine to form LC3phosphatidylethanolamine conjugate (LC3-II), which is recruited to autophagosomal membranes that engulf the damaged proteins. If nuclear receptor coactivator 4 (NCOA4) is involved in ferritin delivery to autophagosome, the proteolytic process is called ferritinophagy [7]. The dysregulation of ferritinophagy leads to change in LIP and correlates many diseases $[8,9]$. Iron chelators can lead to ferritin degradation and the route of its degradation is dependent on the specificity of iron chelators [10-12]. A cancer cell has higher iron demand than normal cell; reduction of iron availability will be a favor to inhibit cancer cell proliferation. One of the mechanisms of iron chelators is a disturbance of iron homeostasis [13-17], in addition, iron chelators can also induce apoptosis, autophagy, and inactivate ribonucleotide reductase. Therefore, iron chelation has been considered as a promising strategy in cancer therapy. Although a lot of works related to iron chelators have been performed and some details in the mechanism were gained, many puzzled issues remain to be solved. Currently, only DFO can induce ferritin degradation (ferritinophagy) in lysosomes via a specific cargo, NCOA4 [7, 18], but whether other iron chelators have a similar action in inducing ferritinophagy was poorly studied. Secondly, the correlation between ferritinophagy and antiproliferative action induced by iron chelators remains to be determined. Furthermore, the relation between the redox property of an iron chelate and antiproliferative action of the ligand during ferritinophagy is poorly understood. Solving the above puzzled issues will enrich our knowledge in cancer chelation therapy. We speculate that the antiproliferative effect of iron chelator might involve ferritinophagy, and redox property of the iron chelate may be a crucial contributor in growth inhibition. In this study, we presented a study of a novel iron chelator, DpdtC (di-2pyridylketone hydrazone dithiocarbamate), on iron mobilization and proliferation, revealing that the occurrence of ferritinophagy was one of the crucial sources for ROS generation and partly contributed to its antitumor activity.

\section{Results}

2.1. DpdtC Mobilized Iron from Ferritin. Previously, we reported DpdtC having the ability in catalase inhibition that may partly contribute to the ROS production [19], which hinted that a Fenton reaction might occur, contributing its antiproliferative action. To extend our knowledge related to the compound (see Supplementary Materials, Figure S1a), in the present study, we assessed the metal chelating ability of DpdtC with iron. And the stoichiometric ratio between DpdtC and ferric chloride was determined by Job's method of continuous variations (see Supplementary Materials). As shown in Figure S1, a new peak around $404 \mathrm{~nm}$ was indicative of complex formation of the DpdtC with iron (III)
(Figure S1c). Accordingly, the composition of the iron complex was also determined (Figure S1d). Similarly, DpdtC could also chelate ferric iron in a similar manner (see Supplementary Materials, Figure S1b). Next, we tested whether DpdtC as like other tridentate chelators could mobilize iron from ferritin; to this end, a purified ferritin was normally used in the assay. Because micromolar concentration required in DpdtC induced growth inhibition (see Section 2.2), thus a low concentration was chosen in the assay of iron mobilization (direct extraction by chelators generally required much higher concentration (3.5-100 mM) to diffuse the $\mathrm{Fe}$ (III)-chelate out of the ferritin shell) [20]. To monitor the iron release from ferritin, the MLCT (metal-toligand charge-transfer) absorption (at $\sim 404 \mathrm{~nm}$ ) that corresponded to $\mathrm{Fe}(\mathrm{III})$-DpdtC complexes was measured during the time course (Figure 1(a)). As shown in Figure 1(a), the absorbance intensity of ferritin at $350 \mathrm{~nm}$ was decreased, but the absorbance at 404 was increased with increasing incubation time, their changes are shown in the insert (Figure 1(b)). To determine whether superoxide radical was involved in the iron mobilization, the superoxide dismutase (SOD) was added, showing that SOD inhibited significantly the iron release, thus indicating the involvement of reactive oxygen species (ROS) in the process of iron removal from ferritin (Figure 1(c)), implying an oxygen-catalytic iron mobilization might be involved, which was similar to that previously reported $[20,21]$.

2.2. The Antiproliferative Activity of the DpdtC. It has been reported that some iron chelators displayed significant proliferation inhibition against tumor cell lines, thus the effect of DpdtC on the proliferation of HepG2 and Bel-7402 cell lines was evaluated based on MTT method. The dose-response curves are depicted in Figure 2. As expected, DpdtC had a significant growth inhibition for HepG2 and Bel-7402 cells $\left(\mathrm{IC}_{50}: 0.4 \pm 0.2\right.$ for HepG2 and $3.5 \pm 0.3 \mu \mathrm{M}$ for Bel-7402). To determine the cytotoxicity of DpdtC to a normal cell, the normal human hepatic cell line LO2 was used for comparison. Figure S2 showed that DpdtC also exhibited a significant growth inhibition against the normal human hepatic cell, with $\mathrm{IC}_{50}=3.15 \pm 0.24 \mu \mathrm{M}$, indicating that the selectivity of the agent was not obvious even greater than that of HepG2 cell in $\mathrm{IC}_{50}$ value. Since that DpdtC was able to chelate iron, the effect of iron ion on the proliferative action was also determined; unexpectedly, the iron (II) addition (equal mole) significantly attenuated the antiproliferative activity of DpdtC against HepG2 cell (Figure 2(b)), but the addition of copper ion significantly enhanced its activity (data not shown), indicating that iron (copper) ions could affect the manner of action of DpdtC due to its chelating ability.

2.3. DpdtC Induced ROS Generation. It has been well documented that most therapeutic drugs in the mechanism can induce production of reactive oxygen species (ROS). The excellent antiproliferative action of DpdtC spurred us to investigate the underlying mechanism. Thus, the cellular ROS level was measured by flow cytometry after the cells were stained by a ROS dye, dichlorofluorescein (DCF). In 


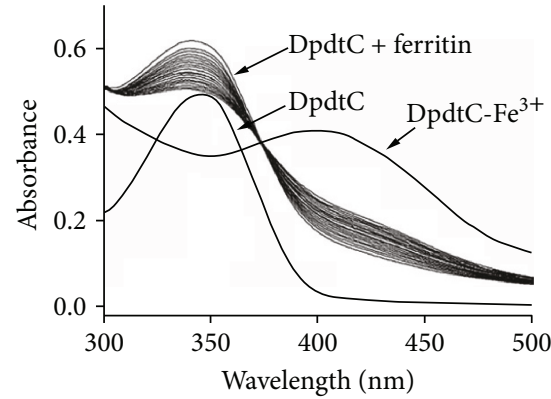

(a)

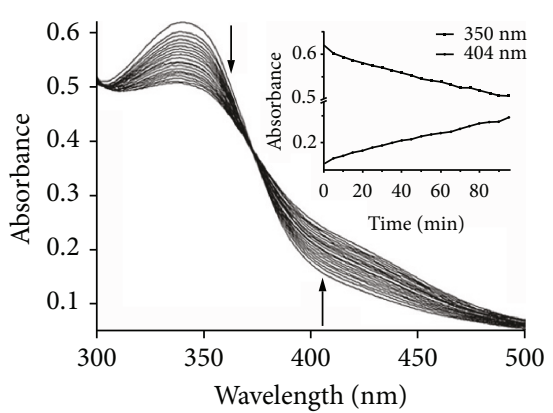

(b)

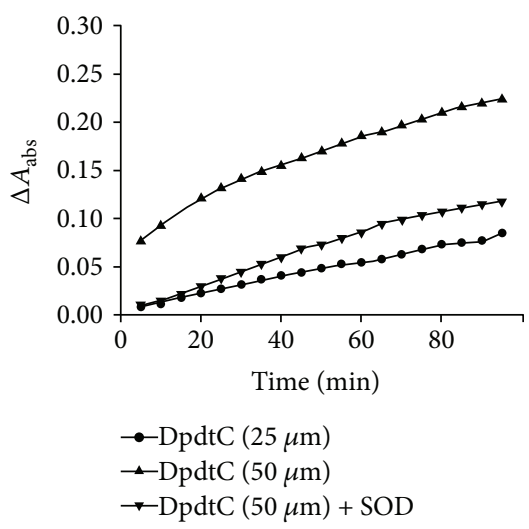

(c)

Figure 1: DpdtC induced iron mobilization from ferritin. (a) The absorbance spectrum of DpdtC and its iron complex; the profiles of iron extracting from ferritin by DpdtC were also included; (b) the spectral changes of ferritin during addition of DpdtC. The insert showed the absorbance changes at specific wavelengths (350 and $404 \mathrm{~nm}$ ); (c) iron mobilization from ferritin by different concentrations of DpdtC was assayed by measuring the absorbance difference at $404 \mathrm{~nm}$, and effect of SOD on iron mobilization was conducted in a similar way.

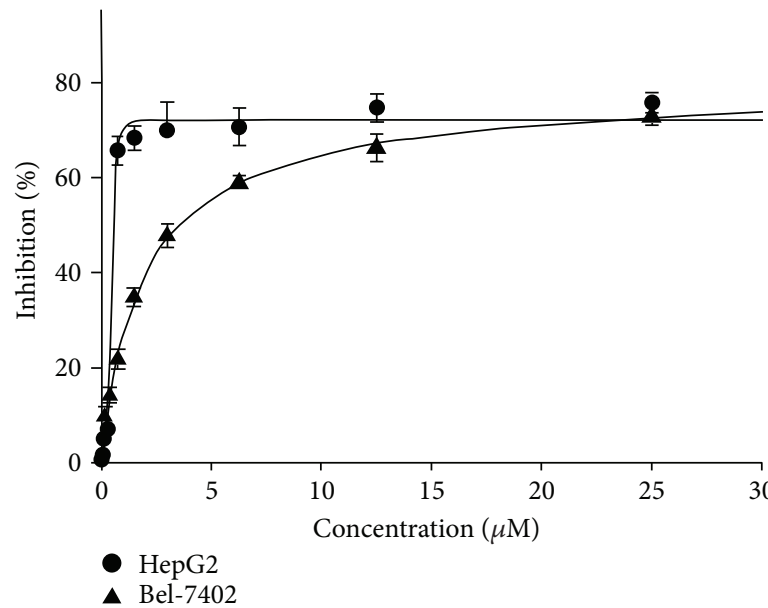

(a)

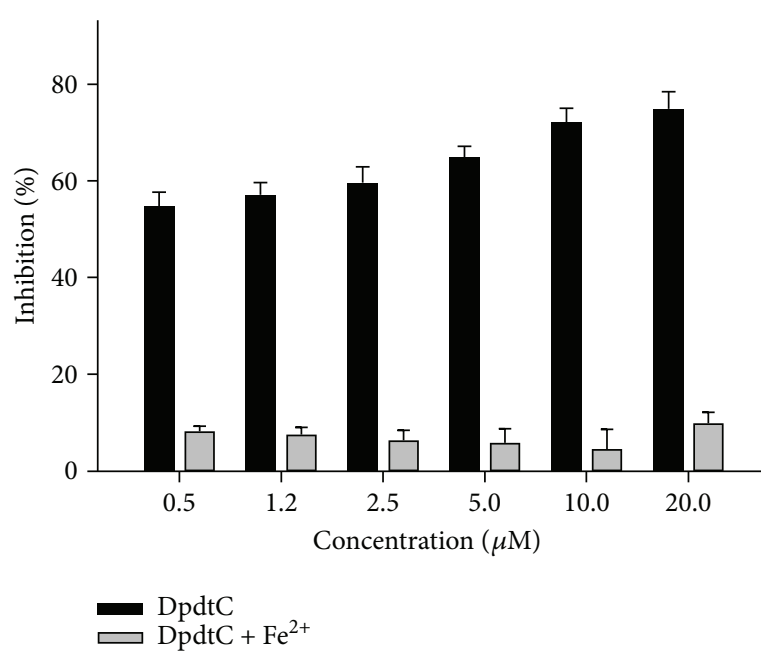

(b)

FIGURE 2: Proliferation inhibition of DpdtC and its iron complex against hepatoma carcinoma cell lines: (a) proliferation inhibition of DpdtC against HepG2 and Bel-7402 cell lines; (b) antiproliferative action of DpdtC against HepG2 cell was attenuated by the addition of iron.

view of higher inhibitory effect on HepG2 cell, the assay was only conducted on this cell line. As shown in Figure 3, the populations in higher fluorescence intensities significantly increased by $21 \sim 34 \%$ after exposure of DpdtC to the cells for $12 \mathrm{~h}$ (a fluctuated pattern of ROS was observed after
$48 \mathrm{~h}$ incubation, Figure S3), hinting that ROS production was involved in the antiproliferative action.

2.4. DpdtC Exposure Resulted in the Accumulation of Autophagic Vacuole. Normally, ROS production associated 


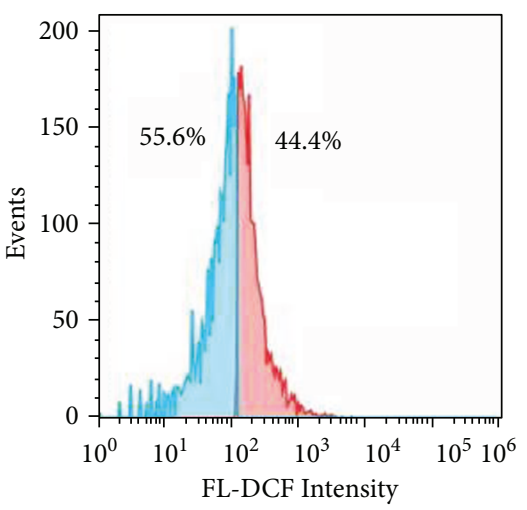

(a)

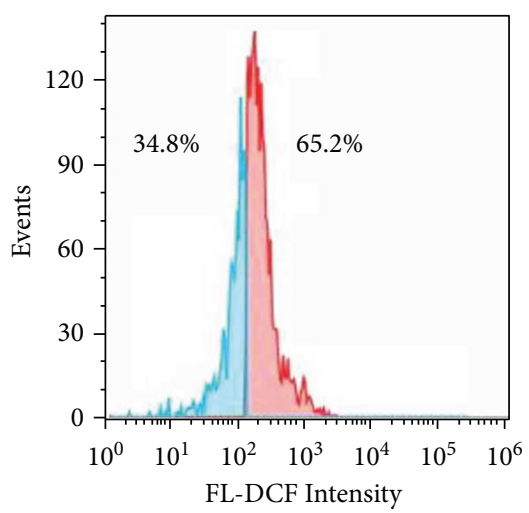

(b)

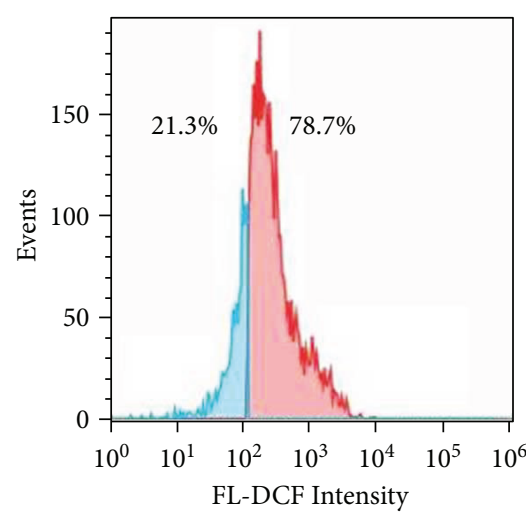

(c)

Figure 3: DpdtC induced ROS production in HepG2 cell. (a) Control; (b) $1 \mu \mathrm{M} \mathrm{DpdtC;} \mathrm{(c)} 2 \mu \mathrm{M}$ DpdtC.

autophagy. Based on ROS assay from HepG2 cell, we speculated that the autophagy might involve DpdtC induced growth inhibition. The formation of acidic vesicular organelles (AVOs) is a characteristic marker in the process of autophagy $[22,23]$. Thus, the AVOs were assayed by acridine orange staining. Figure 4 clearly showed that the fluorescence intensity of granular acridine orange (red in acidic vesicular organelles) increased (Figures 4(b) and $4(c)$ ), implying autophagy occurrence. The addition of an autophagy inhibitor, 3-methyladenine (3-MA), could significantly attenuate the fluorescence intensities of AVOs, supporting above deduction. Interestingly, the addition of iron $\left(\left(\mathrm{NH}_{4}\right)_{2} \mathrm{Fe}\left(\mathrm{SO}_{4}\right)_{2}\right)$ also attenuated the fluorescence intensities of AVOs, indicating that iron as like 3-MA led to decrease in AVOs formation. In addition, iron also attenuated the antiproliferative action of DpdtC (Figure 2(b)). Those implied the alteration of DpdtC action upon addition of iron, and it was related to autophagy (Figures 4(f) and 4(g)).

The alteration in AVOs or autophagosomes was further determined by monodansylcadaverine (MDC) staining via flow cytometry technique [24]. As shown in Figure 5, DpdtC exposure led to $11.5 \%$ increase in higher fluorescence population compared to without treatment of the agent, but the fluorescence intensities were significantly attenuated by the addition of 3-MA (Figures 5(a) and 5(c)), supporting that the accumulation of autophagic vacuole was stemmed from DpdtC stimulus. Similar results were observed in the microscopic analysis (Figure S4). Furthermore, NAC could neutralize the effect of DpdtC (Figure 5(d)), implying that ROS played an important role in autophagy induction.

2.5. DpdtC Exposure Induced Ferritinophagy. The cellular ROS production is mainly from the mitochondrial, while the labile iron pool (LIP) was able to promote the formation of reactive oxygen species (ROS). And LIP and ROS levels were shown to follow similar "rise and fall" patterns [25]. Next, we questioned that the rise in ROS may also indicate higher LIP content after DpdtC exposure to the HepG2 cell and correlate ferritin (FT) degradation. In view of the fact that the FT degradation may occur via autophagy, the changes of cellular ferritin and autophagic marker, LC3 was monitored via immunofluorescence technique. As shown in Figure 6, the upregulated LC3 and downregulated ferritin were observed (Figures 6(a) and 6(f)), indicating that the ferritin degradation was via autophagic proteolysis. To further confirm the above conclusion, the 3-MA, an inhibitor in the formation of autophagic vacuole was added during exposure of DpdtC to the HepG2 cells. As expected, the ferritin degradation was significantly attenuated (Figures $6(\mathrm{~g})$ and 6(i)), confirming that the downregulated ferritin was stemmed from autophagic degradation.

Since the ferritin degradation was via autophagy, the ferritinophagy might occur. To test the hypothesis, the specific carrier for ferritinophagy, NCOA4, and ferritin as well as LC3 were determined by Western blotting. As shown in Figure 7, the ferritin and LC3-I were decreased with increased DpdtC, but the LC3-II and NCOA4 were increased with increased DpdtC, clearly indicating that DpdtC could induce ferritinophagy, which was similar to DFO in ferritinophagy induction [7].

2.6. The DpdtC Induced Change in Lysosomal Membrane Permeability (LMP). Since DpdtC induced ferritinophagy that occurred in lysosomes, the iron release from digested ferritin would synchronously occur. Next, the ferric would be reduced by the reducing agents, which triggered Fenton reaction, producing massive ROS that caused an alteration in LMP. To test the hypothesis, LysoTracker Red that can accumulate within lysosomes was employed to assess the lysosome membrane permeability [13]. As shown in Figure 8, the red fluorescence intensities of HepG2 cells were increased with treatment of DpdtC compared to nontreatment of the cells, indicating that more LysoTracker Red accumulated in lysosomes and LMP was altered (Figure 8(b)). However, the addition of NAC could attenuate the alteration of LMP, hinting that the change correlated to ROS production. Similar situations were observed when addition of 3-MA or chloroquine (Figures $8(\mathrm{~d})$ and 8(f)), indicating that autophagy inhibition could attenuate the alteration in LMP. Interestingly, DFO could also significantly attenuate fluorescence intensities of LysoTracker Red in lysosomes, demonstrating that the Fenton reaction derived from 


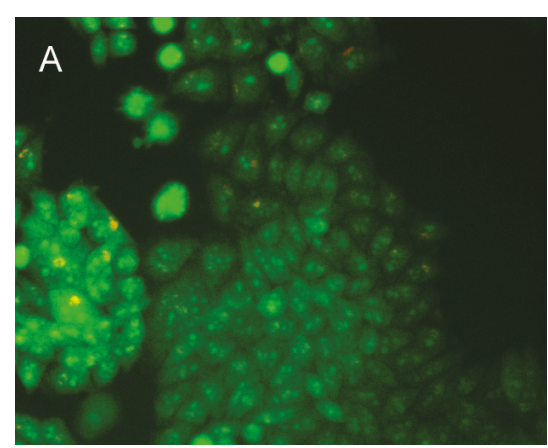

(a)

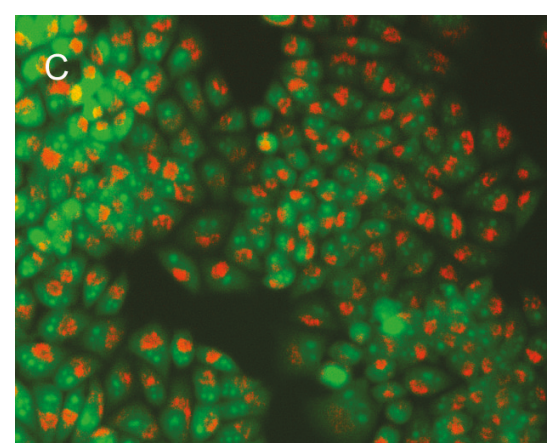

(c)

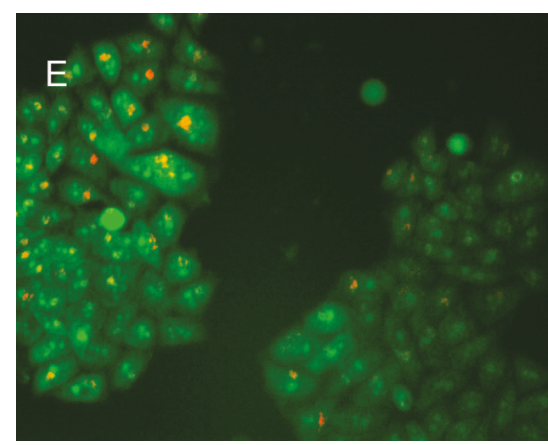

(e)

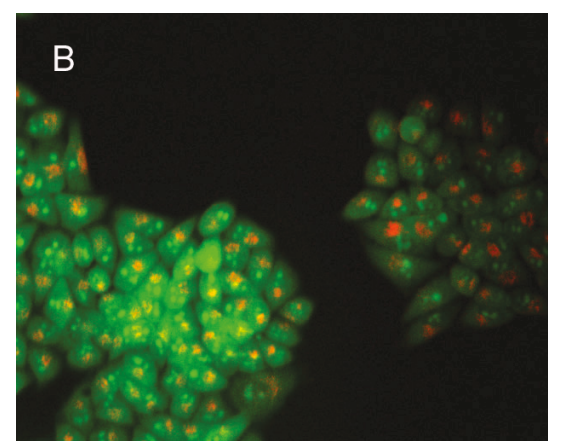

(b)

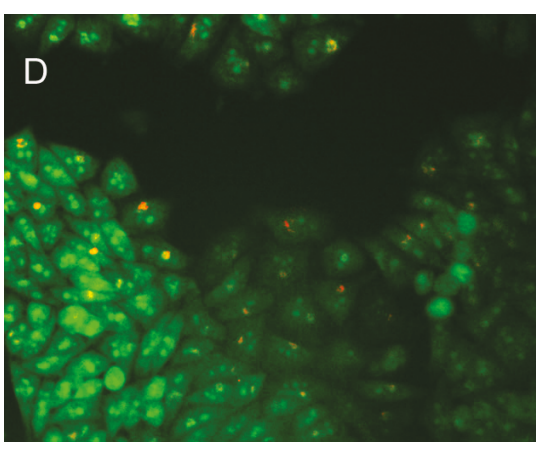

(d)

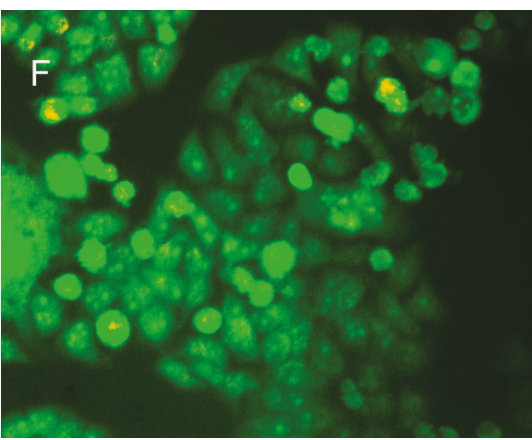

(f)

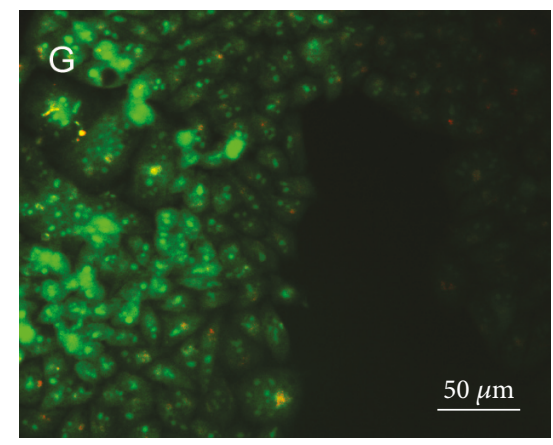

(g)

Figure 4: DpdtC induced formation of acidic vesicular organelles autophagy; the HepG2 cells were treated by the indicated agents for $24 \mathrm{~h}$. (a) Control; (b) $1 \mu \mathrm{M} \mathrm{DpdtC;} \mathrm{(c)} 2 \mu \mathrm{M} \operatorname{DpdtC}$; (d) $1 \mu \mathrm{M} \operatorname{DpdtC}+3-\mathrm{MA}$; (e) $2 \mu \mathrm{M} \operatorname{DpdtC}+3-\mathrm{MA}(2.5 \mathrm{mM}$ ); (f) $1 \mu \mathrm{M}$ $\mathrm{DpdtC}+\mathrm{Fe}^{2+}(1 \mu \mathrm{M}) ;(\mathrm{g}) 2 \mu \mathrm{M}$ DpdtC $+\mathrm{Fe}^{2+}(2 \mu \mathrm{M})$, scale bar: $50 \mu \mathrm{m}$.

ferritinophagy could be quenched by DFO (Figure 8(e)) due to redox inactive DFO-iron chelate. This clearly indicated that lysosomal ROS were stemmed from iron release from ferritinophagy. The quantification analysis of fluorescence changes in different groups was shown in Figure S5.
2.7. Upregulation of TRPML1 Correlated to Autophagy. TRPML1 (transient receptor potential cation channel, mucolipin subfamily, member 1) is a cation-permeable channel that is predominantly localized on the membranes of late endosomes and lysosomes [26]. TRPML1 may mediate both 


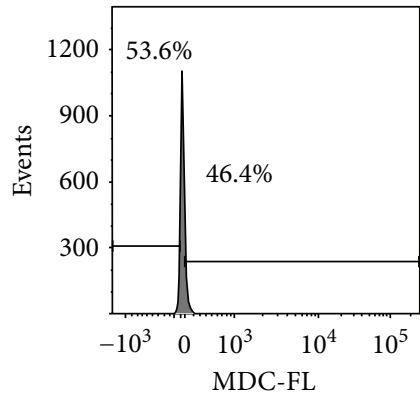

(a)

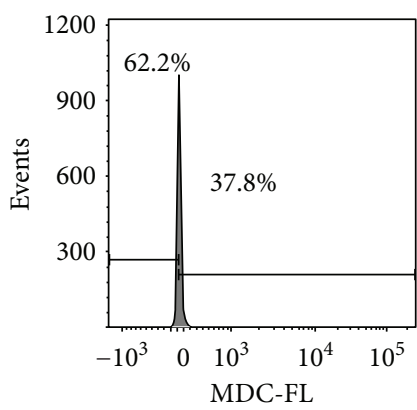

(c)

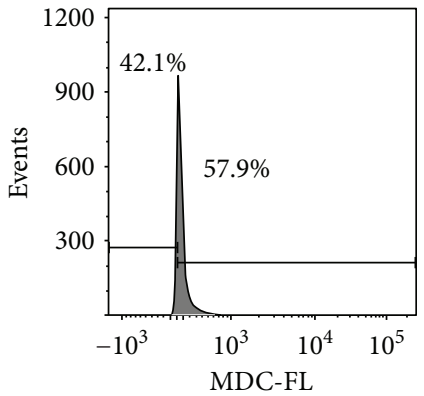

(b)

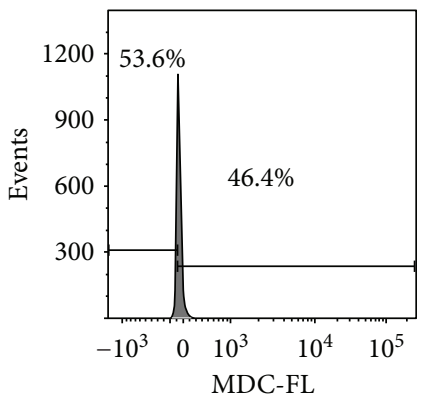

(d)

FIgURE 5: MDC staining for determination of autophagosomes induced by DpdtC. A similar protocol was used in the determination of AVOs via acridine orange staining, but in this assay, MDC and flow cytometry were used. (a) Control; (b) $2 \mu \mathrm{M}$ DpdtC; (c) $2 \mu \mathrm{M}$ DpdtC + 3-MA(1.5 mM); (d) $2 \mu \mathrm{M} \mathrm{DpdtC}+\mathrm{NAC}(1.5 \mathrm{mM})$.

the release of $\mathrm{Ca}^{2+}$ and heavy metal $\mathrm{Fe}^{2+/} \mathrm{Zn}^{2+}$ ions into the cytosol from the lysosomes [27]; thus, TRPML1 was considered as a lysosomal ROS sensor [28]. Next, we assayed TRPML1 expression when DpdtC was exposed to the HepG2 cells. As expected, DpdtC led to upregulation of TRPML1 (Figure 9(a)), but downregulation of TRPML1 was observed by addition of 3-MA or NAC (Figure 9(a)), indicating that the lysosomal ROS was stemmed from the occurrence of ferritinophagy, in consistent with the changes in LC3-II. The quantitative analyses of TRPML1 and LC3-II are shown in Figure 9(b).

2.8. Lipid Peroxidation Occurred When DpdtC Was Exposed to HepG2 Cell. As mentioned above, the cellular ROS were increased when DpdtC was exposed to the HepG2 cells. The intracellular ROS at least partly originated from lysosome due to the occurrence of ferritinophagy that may involve Fenton-like reactions [29]. Excess ROS lead to damage biological macromolecules, including lipid peroxidation. To test this hypothesis, a lipid peroxidation assay was performed as described previously [30]. As shown in Figure 10, DpdtC significantly enhanced lipid peroxidation in a concentrationdependent manner, but the addition of 3-MA drastically attenuated the lipid peroxidation, consistent with the results from ferritinophagy.

\section{Discussion}

A widely accepted concept is that iron chelators can coordinate iron from cytosolic labile iron pool (LIP), leading to iron depletion that provokes the association of iron response proteins with iron response element (IRE) in UTRs (untranslated regions) of various mRNAs whose products are involved in iron metabolism [31]. Iron chelators are also capable to mobilize iron from ferritin in vitro, leading to form an iron poor ferritin, but the evidence of iron mobilization by chelator in cellular level is still lacking. A recent study showed that ferritin-Fe mobilization does not occur through changes in cellular concentrations of reducing/chelating agents but by the coordinated movement of ferritin and DMT1 to lysosomes [32], which causes ferritin degradation in the lysosome. Iron chelators can sequester iron, but the redox potentials of the resulting complexes (iron) varied with association constant. The iron chelate can serve as either a reductant or an oxidant, depending on the redox potential of its opponent encountered; therefore, iron chelator has two sides, either as an antioxidant or as a prooxidant. However, in vivo (or cellular level) this situation is more complicated due to different cellular locations of the iron chelates. Generally, iron chelators function as either an antioxidant or a prooxidant, depending on the redox nature of iron complex formed $[33,34]$; however, the direct correlation between ROS production and viability remains to be determined. Many iron chelators, such as deferoxamine (DFO), deferiprone, and deferasirox, caused ferritin degradation, but only DFO-induced ferritin loss was prevented by chloroquine treatment, indicating that DFO-induced proteolysis occurred in lysosomes; the others led to ferritin degradation in proteomes, which depended on the specificity of the chelators $[10,11]$. In addition, iron chelators also displayed excellent antitumor activities [35]; the representative 


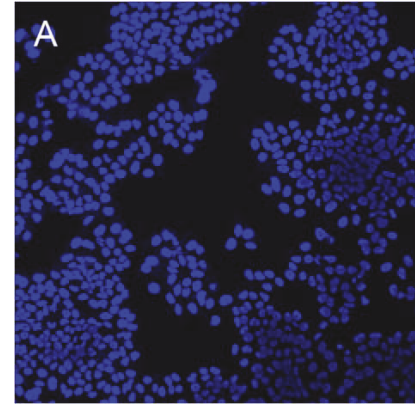

(a)

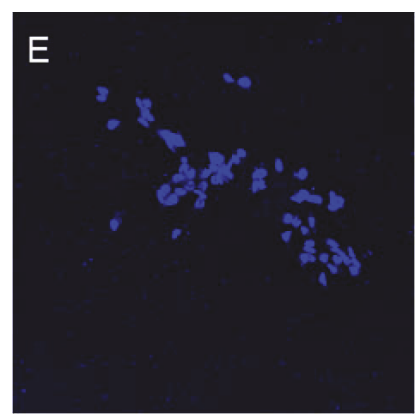

(e)

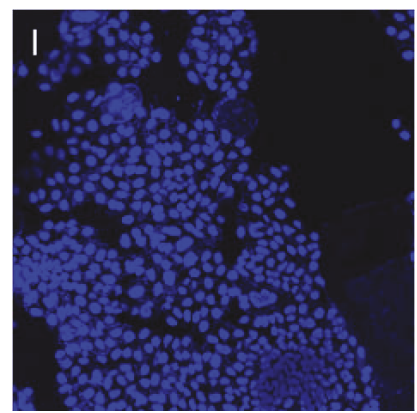

(i)

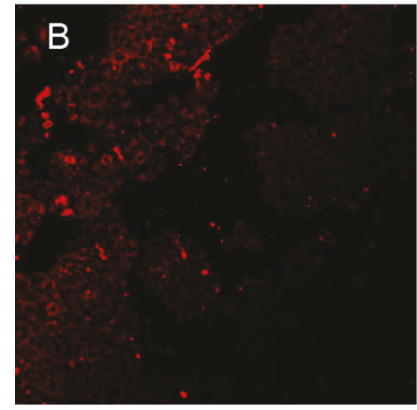

(b)

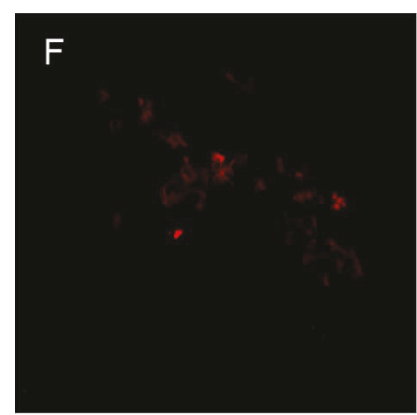

(f)

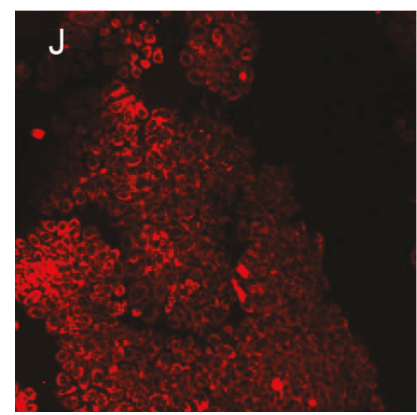

(j)

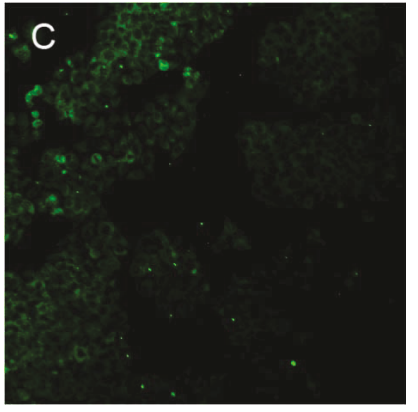

(c)

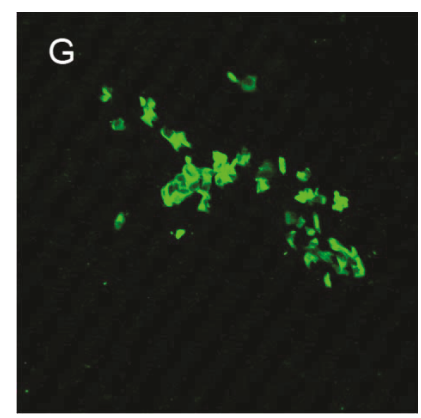

(g)

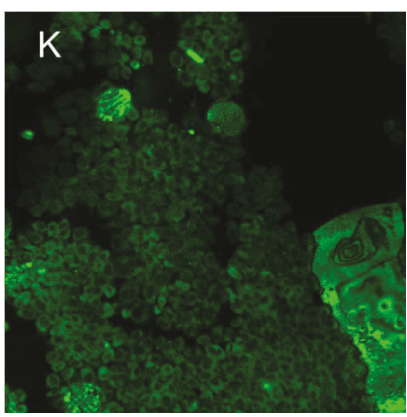

(k)

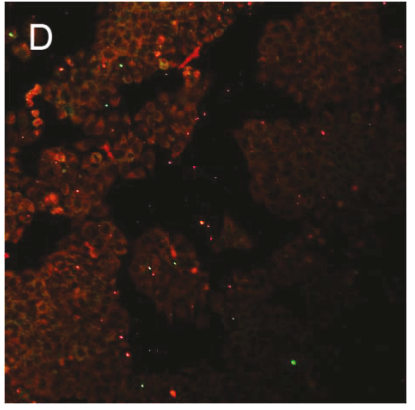

(d)

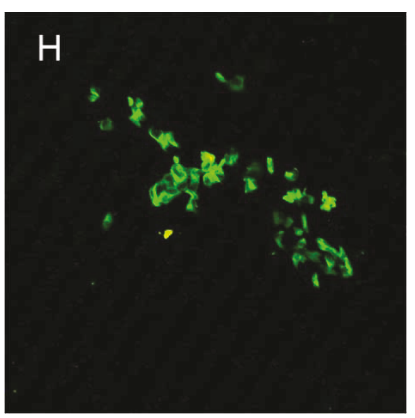

(h)

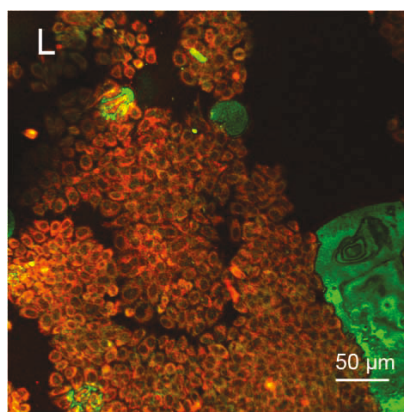

(1)

FIGURE 6: DpdtC induced ferritin autophagy (ferritinophagy). The nuclei stained by DAPI in blue, ferritin labeled in red, and LC3 labeled in green. (a-d) Control group; (a) nuclei in blue; (b) ferritin in red; (c) LC3 in green; (d) merging of ferritin with LC3. (e-h) DpdtC treated group: (e) nuclei in blue; (f) ferritin in red; (g) LC3 in green; (h) merging of ferritin with LC3. (i-l) DpdtC combined with 3-MA group: (i) nuclei in blue; (j) ferritin in red; (k) LC3 in green; (l) merging of ferritin with LC3, scale bar: $50 \mu \mathrm{m}$.

chelators are heterocyclic carboxaldehyde thiosemicarbazones (Dp44mT), analogs of pyridoxal isonicotinoyl hydrazine (PIH), tachpyridine, o-trensox, and other natural products (DFO, desferrithiocin), and many of them are at various stages of clinical trials $[36,37]$. However, some disadvantages of iron chelators such as shorter plasma half-life (DFO) and high toxicity in the kidney and neurological problems have motivated the scientific community to find new iron ligands $[35,38]$. In the present study, we presented the investigation of a novel dithiocarbamate derivative, DpdtC, on the characteristic of iron mobilization and antiproliferative activity against hepatoma carcinoma cell lines (Figures 1 and 2). The excellent antiproliferative action of DpdtC promoted us to determine the underlying mechanism (Figure 2). Data revealed that DpdtC, as other iron chelators, could induce ROS production (Figure 3) [35], triggering either physiologic or cytotoxic autophagy. Due to multiple steps in autophagy occurrence, an important step is autophagosome formation; thus, the alteration in autophagic vacuoles when DpdtC was exposed to HepG2 cell by both acridine orange and MDC staining was investigated. As expected, the autophagic vacuoles increased after exposure of DpdtC to HepG2 cell but significantly decreased when combined with either NAC or 3-MA (Figures 4 and 5 and Figure S3), indicating that the manner of action of DpdtC involved autophagy and ROS production. Many chelators can induce ferritin degradation that may occur in lysosomes, thus the level of cytoplasmic ferritin was determined. As expected, a decrease in ferritin and an increase in LC3-II were observed (Figure 7), implying that the ferritin degradation occurred in lysosomes. Since ferritin degradation was associated with autophagy, this process might be also involved in ferritinophagy. To support this hypothesis, the status of specific cargo for ferritinophagy, NCOA4, required to be determined (Figure 7). As expected, accompanied by downregulated ferritin an upregulated NCOA4 was 

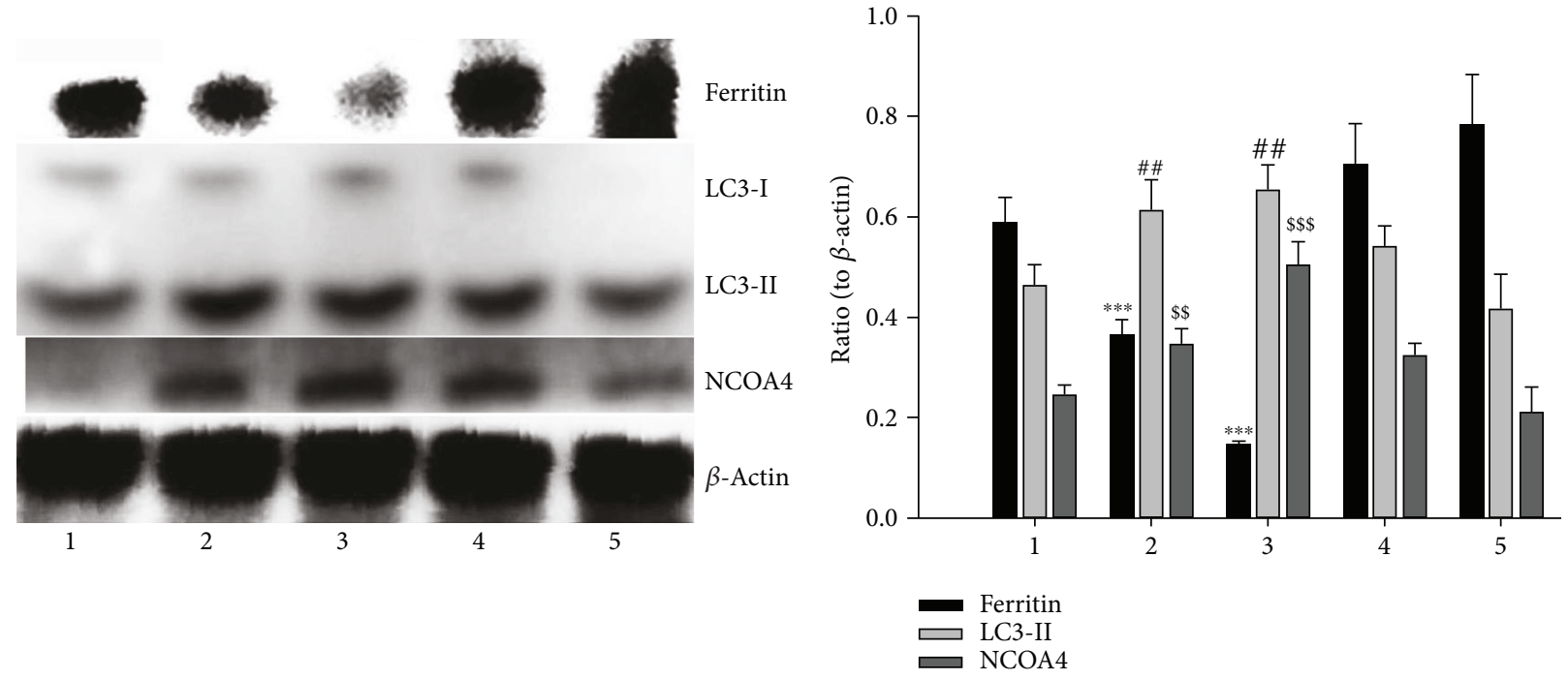

(a)

(b)

Figure 7: DpdtC induced ferritinophagy. (a) Western blot analysis; (b) quantification analysis. The data were obtained from three independent Western blots based on comparison of the investigated protein with $\beta$-actin. (1) Control (water); (2) $1 \mu \mathrm{M} \mathrm{DpdtC}$; (3) $2 \mu \mathrm{M}$ DpdtC; (4) $1 \mu \mathrm{M}$ DpdtC + 3-MA (1.5 mM); (5) $2 \mu \mathrm{M} \operatorname{DpdtC}+3-\mathrm{MA}(1.5 \mathrm{mM}) .\left({ }^{* * *, \$ \$} p<0.01\right.$; \#\#,\$p<0.05; one-way ANOVA).

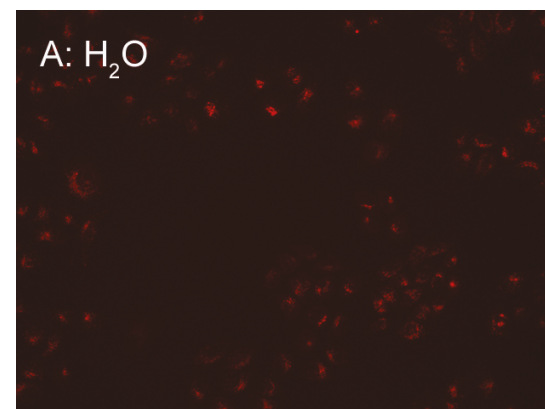

(a)

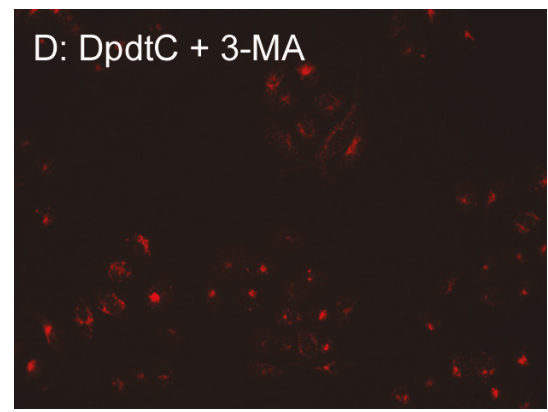

(d)

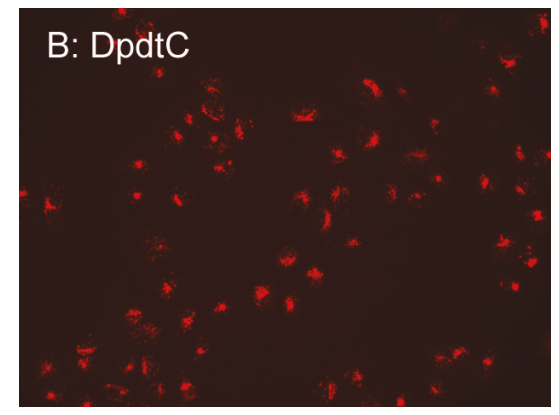

(b)

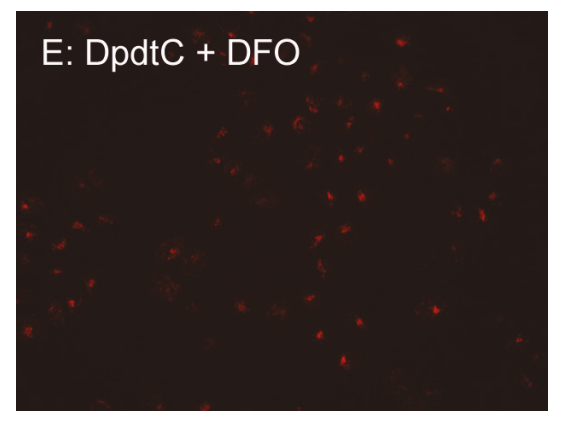

(e)

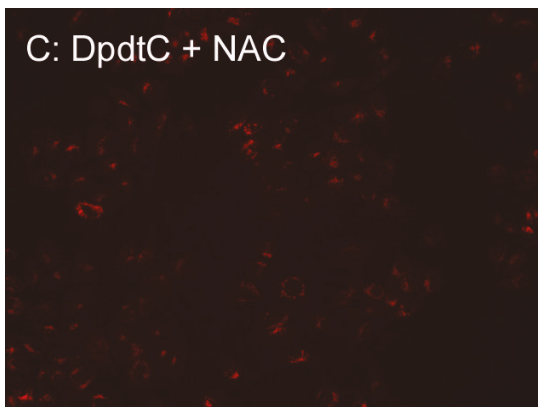

(c)

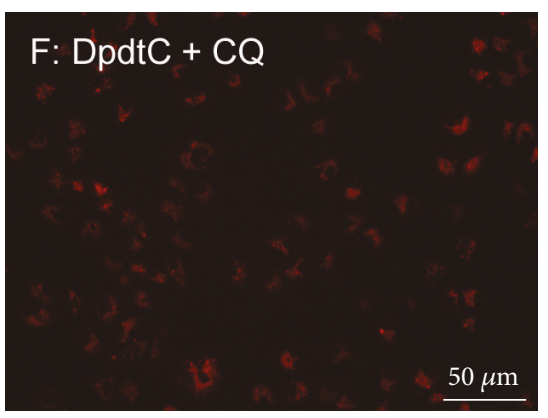

(f)

FIGURE 8: DpdtC induced change in lysosomal membrane permeability (LMP). LysoTracker Red stained HepG2 cells: (a) control; (b) $1.0 \mu \mathrm{M}$ DpdtC; (c) $1.0 \mu \mathrm{M} \mathrm{DpdtC}+\mathrm{NAC}(1.5 \mathrm{mM})$. (d) $1.0 \mu \mathrm{M} \mathrm{DpdtC}+3-\mathrm{MA}(1.5 \mathrm{mM}) ;$ (e) $1.0 \mu \mathrm{M} \mathrm{DpdtC}+\mathrm{DFO}(50 \mu \mathrm{M}) ;(\mathrm{f}) 1.0 \mu \mathrm{M} \mathrm{DpdtC}+\mathrm{CQ}$ $(50 \mu \mathrm{M})$. Scale bar: $50 \mu \mathrm{m}$.

founded, indicating that ferritinophagy indeed occurred. This is the first report that DpdtC, a dithiocarbamate derivative, induced ferritinophagy except DFO [7]. Owing to the occurrence of ferritinophagy, the abundance of lysosomal iron increased; consequently, an increase in LIP, oxidative stress, and cell death may come up [39]. The consequence of ferritinophagy inevitably led to lysosomal destruction.
Figure 8 showed that more LysoTracker Red dyes were accumulated in lysosomes after exposure of DpdtC to HepG2 cells, but this accumulation could be attenuated by the addition of NAC (Figure 8(c)), 3-MA, or chloroquine, indicating that the damage of lysosomal membrane (or integrity) was caused by ROS and ferritinophagy $[21,40]$. It was imagined that the ferric iron was liberated from digested ferritin and 


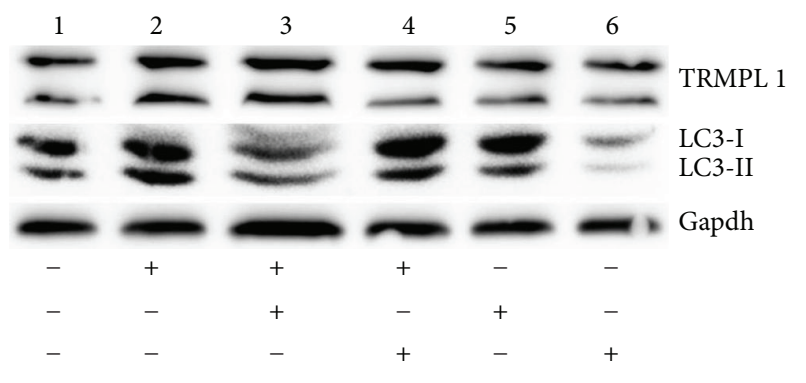

(a)

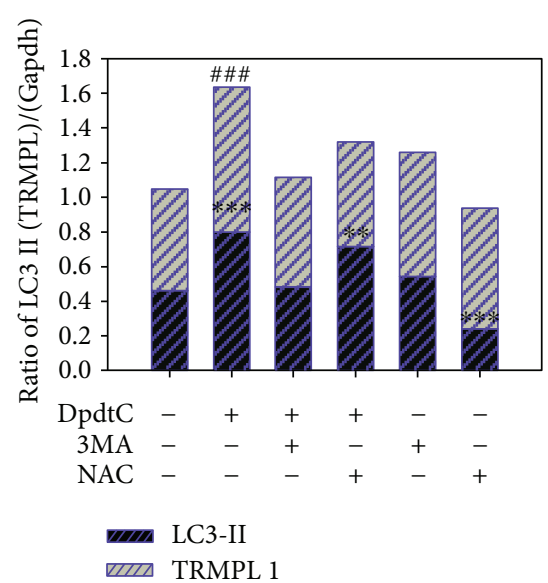

(b)

FIgure 9: The effect of lysosomal ROS on autophagy and TRMPL1. A: Western blot analysis; B: quantification analysis. The data were obtained from three independent Western blots based on comparison of the investigated protein with Gapdh (1) control; (2) $1 \mu \mathrm{M}$ DpdtC; (3) $1 \mu \mathrm{M} \mathrm{DpdtC}+1.5 \mathrm{mM} 3-\mathrm{MA}$; (4) $1 \mu \mathrm{M}$ DpdtC+1.5 mM NAC; (5) $1.5 \mathrm{mM} \mathrm{3-MA;} \mathrm{(6)} 1.5 \mathrm{mM} \mathrm{NAC.}\left({ }^{* * *}\right.$,\#\#\# $p<0.01$; ${ }^{* *} p<0.05$; one-way ANOVA).

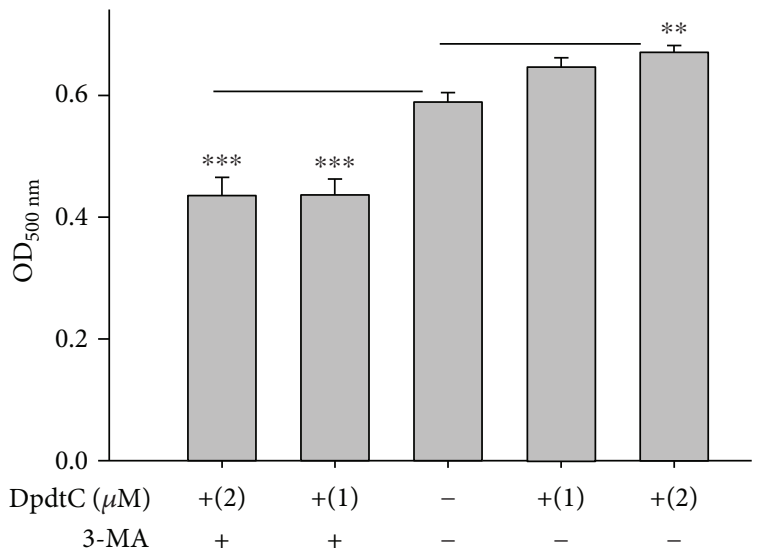

FIgURE 10: A lysosomal ROS caused lipid peroxidation. The absorbance at $500 \mathrm{~nm}$ represents the peroxidative degree of lipid; clearly, DpdtC induced lipid peroxidation, but 3-MA could attenuate the oxidative effect, indicating that the lipid peroxidation was related to autophagy. $\left({ }^{* * *} p<0.01 ;{ }^{* *} p<0.05\right.$, one-way ANOVA).

reduced further by the endosomal ferrireductase Steap3 in the acidified lysosome [41]; the resulting ferrous ion triggered Fenton reaction. To further confirm that the lysosomal ROS were stemmed from Fenton reaction, DFO, an iron chelator (lysophilic chelator) that can protect the lysosome membrane from oxidative damage was employed [42]. As shown in Figure 8(e), DFO efficiently decreased the accumulation of LysoTracker Red dyes, quenching Fenton reaction. During ferritinophagy, iron leaking from the lysosomes to the cytosol may also occur either via DMT-1 or lysosomal iron channel TRPML1 that was considered as a lysosomal ROS sensor $[43,44]$; therefore, the expression of TRPML1 reflected the status of lysosomal ROS and iron leaking. As expected (Figure 9), an upregulated TRPML1 was observed, but the addition of NAC could downregulate TRPML1, indicating that the cell death induced by DpdtC was a lysosomal
ROS dependent. Excess ROS leading to lipid peroxidation, proteins, and DNA damage has been well documented [45]; the lipid peroxidation correlated with excess ROS production. Figure 10 showed that DpdtC induced an increase in lipid peroxidation, but the peroxidation could be attenuated by the addition of 3-MA, implying that autophagy was a crucial contributor in growth inhibition [46]. It should be noted that the autophagy (ferritinophagy) and growth inhibition induced by DpdtC could be eliminated by addition of iron ion (Figures 2 and 4); a reasonable explanation for this may be due to ability loss in moving ferritin to lysosome upon DpdtC chelates iron [32], and a much higher cytoplasmic DpdtC concentration than iron in LIP may ensure its ability in ferritinophagy induction.

Taken together, the growth inhibition against hepatic cancer induced by DpdtC was partly correlated with ferritinophagy that triggered lysosomal ROS production via Fenton reaction and iron release from lysosomes to cytoplasm. Obviously, the occurrence of ferritinophagy was prerequisite; secondly, the redox activity of iron chelate (redox property) and location of chelator in the cell may be crucial factors, which determined subsequent biological effects, such as growth inhibition. However, insight into the correlation between redox nature of iron chelate and induced growth inhibition requires more studies in the future due to the diversity of iron chelators in structure and complex interactions with biological molecules.

\section{Materials and Methods}

4.1. Materials. All reactants and solvents were analytical reagents (AR) grade. 3-(4,5-dimethylthiazol-2-yl)-2,5-diphenyltetrazolium bromide (MTT), monodansylcadaverine (MDC), 3-methyladenine (3-MA), chloroquine, dichlorofluorescein ( $\left.\mathrm{H}_{2} \mathrm{DCF}-\mathrm{DA}\right)$, deferoxamine (DFO), 4',6-diamidino-2-phenylindole (DAPI), Roswell Park Memorial Institute (RPMI) 1640, Horse spleen ferritin, and other 
chemicals were purchased from Sigma-Aldrich. LC3 and ferritin antibody were obtained from Proteintech Group (Wuhan, China). Nuclear receptor coactivator 4 (NCOA4) antibody was purchased from Boster Biological Technology Co. Ltd. (Wuhan, China), and superoxide dismutase (SOD) was obtained from Beyotime Biotechnology (Beijing, China). Transient receptor potential cation channel, mucolipin subfamily, member 1 (TRPML1) antibody was purchased from Abcam (Shanghai, China). Secondary antibodies (or fluorescence labeled) were obtained from EarthOx, LLC (San Francisco, USA).

4.2. Iron Mobilization by DpdtC. The iron release experiments were conducted in $0.05 \mathrm{M}$ Tris- $\mathrm{HCl}, 50 \mathrm{mM}$ $\mathrm{NaCl}, \mathrm{pH} 7.4,10 \mu \mathrm{l}$ ferritin $(12.5 \mathrm{mg} / \mathrm{ml})$, and varied concentration of DpdtC (or with 3 unit SOD) in a total of $1 \mathrm{ml} \mathrm{vol-}$ ume. The kinetics of iron release from ferritin was monitored by the increase in the characteristic of the MLCT (metal-toligand charge-transfer) absorption bands of the corresponding iron (II)-chelate complexes (404 nm for DpdtC). The absorbance was measured every $5 \mathrm{~min}$ on a Shimadzu UV-2450 spectrophotometer with thermostatic circulating device at $37^{\circ} \mathrm{C}$.

4.3. Cytotoxicity Assay (MTT Assay). The stock solution of DpdtC $(10 \mathrm{mM})$ was prepared in autoclaved deionized water and diluted to the required concentration with culture when used. HepG2 cells (or Bel-7402, LO2) were cultured in RPMI 1640 medium supplemented with $10 \%$ fetal calf serum (FCS) and antibiotics. The cells in exponential phase were collected and seeded equivalently into a 96-well plate; next, the varied DpdtC (or in the presence of equivalent iron (II) salt) was added after the cells adhered. Following $48 \mathrm{~h}$ incubation at $37^{\circ} \mathrm{C}$ in a humidified atmosphere of $5 \% \mathrm{CO}_{2}, 10 \mu \mathrm{l}$ MTT solution $(5 \mathrm{mg} / \mathrm{ml})$ was added and further incubated for $4 \mathrm{~h}$. The cell culture was removed by aspiration, and $100 \mu \mathrm{l}$ DMSO was added in each well to dissolve the formazan crystals. The measurement of absorbance of the solution that was related to the number of live cells was performed on a microplate reader (MK3, Thermo Scientific) at $570 \mathrm{~nm}$. Percent growth inhibition was defined as percent absorbance inhibition within appropriate absorbance in each cell line. The same assay was performed in triplet.

4.4. Flow Cytometry Analysis of Cellular ROS. Similar to MTT assay, the HepG2 cells were treated by either DpdtC or in combined with 3-MA (or NAC) for $24 \mathrm{~h}$. The cells were collected by centrifugation after trypsinization. Following by PBS washing, the cells pellet was resuspended in $\mathrm{H}_{2}$ DCF-DA containing serum-free culture medium and incubated for $30 \mathrm{~min}$. Next, after removing the $\mathrm{H}_{2}$ DCF-DA contained medium by centrifugation and washing with PBS, the cells were finally resuspended in PBS. The intracellular ROS assay was performed on a flow cytometer (Becton-Dickinson, USA).

4.5. Flow Cytometric and Microscopic Analyses of Autophagic Vacuoles. Cells were seeded into a 6-well plate and treated as described above for the cell viability assay. The cells were treated with either the agent alone $(1.0$ or $2.0 \mu \mathrm{M})$ or a combination with 3 - MA $(1.5 \mathrm{mM})$ or NAC $(1.5 \mathrm{mM}))$ for $24 \mathrm{~h}$. Then, the cell culture was removed, following PBS washing, trypsin digestion; finally, the MDC $(50 \mu \mathrm{M})$ were added as described previously [47]. The stained cells were subjected to flow cytometric analysis (Becton-Dickinson, USA).

4.6. Immunofluorescence Analysis. HepG2 cells were first cultured in a 6-well plate with a cover glass overnight. Following DpdtC treatment for $24 \mathrm{~h}$, the cells were first fixed with $4 \%$ paraformaldehyde in $\mathrm{PBS}$ for $15 \mathrm{~min}$ at $37^{\circ} \mathrm{C}$, and then permeabilized with $0.2 \%$ Triton X-100 in PBS for $10 \mathrm{~min}$. After blocking with 1\% BSA in PBS for $30 \mathrm{~min}$, the cells were incubated with LC3 and ferritin (H chain) primary antibody based on dilution recommended by the company; at $4^{\circ} \mathrm{C}$ the plate was shaken overnight. Next, removing the primary antibodies and washing with PBS, the cells were further incubated with fluorescence-labeled secondary antibody for $3 \mathrm{~h}$ at room temperature. After removing the secondary antibody, the cells were further counterstained with DAPI. Finally, a confocal laser scanning microscope (LSM 410, Zeiss, Jena, Germany) was used to visualize the cells; the representative cells were selected and photographed.

4.7. Autophagy and Lysosomal Membrane Permeability (LMP) Affected by DpdtC. The alteration of LMP was assayed as previously described [13]. For the detection of the acidic cellular compartment, acridine orange (or LysoTracker Red; Invitrogen) was used, which emits bright red fluorescence in acidic vesicles but green fluorescence in the cytoplasm and nucleus. After treatment of the cells with the agent, acridine orange was then added at a final concentration of $1 \mu \mathrm{g} / \mathrm{ml}$ (the concentration of LysoTracker Red, as recommended) for a period of $15 \mathrm{~min}$. Following PBS washing, the fluorescent micrographs were captured using an inverted fluorescence microscope.

4.8. Western Blotting Analysis. Briefly, $1 \times 10^{7}$ HepG2 cells, treated with or without DpdtC, were scraped in lysis buffer (50 mM Tris-HCl, pH 8.0, $150 \mathrm{mM} \mathrm{NaCl}, 1.0 \%$ NP-40, 10\% glycerol, and protease inhibitors) and subjected to sonication, followed by centrifugation at $14,000 \times \mathrm{g}$. The clear supernatant was stored at $-80^{\circ} \mathrm{C}$. Protein concentration was determined using a colorimetric Bio-Rad DC protein assay using the MK3 microplate reader at $570 \mathrm{~nm}$. Proteins $(30 \mu \mathrm{g})$ were separated on a 13 15\% sodium dodecyl sulfate-polyacrylamide gel at $200 \mathrm{~V}$ for $3 \mathrm{~h}$. The separated proteins were subsequently transferred onto a PVDF membrane at $60 \mathrm{~V}$ for $2 \mathrm{~h}$. The membrane was washed three times with Tris-buffered saline (TBS) and then blocked for $2 \mathrm{~h}$ in TBS containing $0.1 \%$ Tween- 20 and $5 \%$ nonfat skimmed milk. The membrane was incubated at $4^{\circ} \mathrm{C}$ overnight with the appropriate primary antibody used at a dilution of $1: 300$ in TBS plus $0.1 \%$ Tween-20 (TBST). The membrane was then washed several times with TBST and subsequently incubated with the appropriate HRPconjugated secondary antibody (1:2000 in TBST) for $1 \mathrm{~h}$ at room temperature. Following washing with TBST, the protein bands were detected using a super sensitive ECL 
solution (Boster Biological Technology Co. Ltd.) and visualized using a Syngene G:BOX imager (Cambridge, United Kingdom). Quantifications of protein bands intensities and fluorescence intensity were performed using ImageJ software.

4.9. Lipid Peroxidation Assay. Lipid peroxidation analysis was performed based on spectrophotometry, in which the ferrous ion is oxidized by lipid hydroperoxides to the ferric ion and subsequently reacts with thiocyanate to form a colored complex [30]. The details were as previously described [48]. Briefly, the trypsinized cells were collected and treated with the DpdtC for $12 \mathrm{~h}$; the supernatant was removed by centrifugation and washed with PBS. The peroxidized lipid was extracted using deoxygenated $\mathrm{CHCl}_{3} / \mathrm{MeOH}(2: 1, v / v$ mixture, $1000 \mu \mathrm{l}$ ), and the lipids were transferred to a $5 \mathrm{ml}$ volumetric flask, which contained $100 \mu$ l of ferrous sulfate $(0.2 \mathrm{M} \mathrm{HCl})$ and $100 \mu \mathrm{l}$ of $3 \%$ deoxygenated thiocyanate (methanol) for $60 \mathrm{~min}$. Finally, deoxygenated $\mathrm{CHCl}_{3} / \mathrm{MeOH}$ solvents were added to the given volume. The absorbances at $500 \mathrm{~nm}$ were measured using a UV-2450 spectrophotometer (Shimadzu Co. Ltd., Suzhou, China). The molar absorptivity of the ferric thiocyanate complex expressed per mol of LOOH was determined to be $58,440 \mathrm{M}^{-1} \mathrm{~cm}^{-1}$ [30].

4.10. Statistical Analysis. Data were analyzed with Prism 5.0 (GraphPad Software Inc., USA). Comparisons were made using a one-way analysis of variance or a two-tailed t-test. Results are presented as the mean \pm SEM. A $p$ value $<0.05$ was considered statistically significant.

\section{Data Availability}

The data used to support the finding of this study are included in the article.

\section{Conflicts of Interest}

The authors declare no conflict of interest.

\section{Authors' Contributions}

Tengfei Huang, Yanjie Sun, and Yongli Li performed the experiments; they contributed equally to this work. Changzheng Li conceived and designed the experiments; Tingting Wang, Yun Fu, and Cuiping Li analyzed the data and generated the figures. Changzheng Li prepared and wrote the paper.

\section{Acknowledgments}

The present study was supported by grants awarded by the Natural Science Foundation of China (no. 21571153), the Henan Science and Technology Agency (no. 152300410118), The Key Research Project Funding Program of Higher Educational Institutions of Henan Province (19A310021), and Innovation team awarded by Sanquan College of Xinxiang Medical University.

\section{Supplementary Materials}

Determination of the molar ratio of DpdtC to iron complex: The reaction stoichiometry between DpdtC and $\left(\mathrm{NH}_{4}\right)_{2} \mathrm{Fe}\left(\mathrm{SO}_{4}\right)_{2}$ was studied by Job's method of continuous variations (13). For this method, different volumes $(0,20,40,60,80,100,120,140,160$, and $180 \mu \mathrm{l})$ of $1 \mathrm{mM}\left(\mathrm{NH}_{4}\right)_{2} \mathrm{Fe}\left(\mathrm{SO}_{4}\right)_{2}$ were added with different volumes $(200,180,160,140,120,100,80,60,40$, and $20 \mu \mathrm{l})$ of $1 \mathrm{mM}$ DpdtC and diluted with water in $5 \mathrm{ml}$ standard volumetric flask. The absorbance was recorded on a Shimadzu UV-2450 spectrophotometer. The absorbance at $404 \mathrm{~nm}$ against the mole fraction of the mole ratio of iron (II) (or DpdtC) was plotted and regressed linearly (SigmaPlot 10.0). As described in experimental section of determination of the molar ratio of DpdtC to $\mathrm{Fe}$ (II) complex, the molar ratio of DpdtC to $\mathrm{Fe}$ (III) was determined by similar method. In this protocol, the ferric chloride was used, and the titration was conducted in acetonitrile (Figure S1). Microscopic analyses of autophagic vacuoles: Cells were seeded into a 6-well plate and treated as described above for the cell viability assay. For morphologic analysis, autophagic vacuoles were labeled with MDC by incubating the cells that treated by either the agent or combination with 3-MA (or NAC) with $50 \mu \mathrm{M} \mathrm{MDC} \mathrm{in} \mathrm{PBS} \mathrm{at} 37^{\circ} \mathrm{C}$ for 10 minutes. After incubation, the cells were washed four times with PBS and immediately analyzed by fluorescence microscopy using an inverted microscope (Shanghai Lengguang Technology Co., Ltd., Shanghai, China) (Figure S3). (Supplementary Materials)

\section{References}

[1] K. Pantopoulos, S. K. Porwal, A. Tartakoff, and L. Devireddy, "Mechanisms of mammalian iron homeostasis," Biochemistry, vol. 51, no. 29, pp. 5705-5724, 2012.

[2] S. J. Dixon and B. R. Stockwell, "The role of iron and reactive oxygen species in cell death," Nature Chemical Biology, vol. 10, no. 1, pp. 9-17, 2014.

[3] M. C. Linder, H. R. Kakavandi, P. Miller, P. L. Wirth, and G. M. Nagel, "Dissociation of ferritins," Archives of Biochemistry and Biophysics, vol. 269, no. 2, pp. 485-496, 1989.

[4] H. N. Munro and M. C. Linder, "Ferritin: structure, biosynthesis, and role in iron metabolism," Physiological Reviews, vol. 58, no. 2, pp. 317-396, 1978.

[5] N. Gálvez, B. Ruiz, R. Cuesta, E. Colacio, and J. M. Domínguez-Vera, "Release of iron from ferritin by acetoand benzohydroxamic acids," Inorganic Chemistry, vol. 44, no. 8, pp. 2706-2709, 2005.

[6] I. de Domenico, M. B. Vaughn, L. Li et al., "Ferroportinmediated mobilization of ferritin iron precedes ferritin degradation by the proteasome," The EMBO Journal, vol. 25, no. 22, pp. 5396-5404, 2006.

[7] J. D. Mancias, X. Wang, S. P. Gygi, J. W. Harper, and A. C. Kimmelman, "Quantitative proteomics identifies NCOA4 as the cargo receptor mediating ferritinophagy," Nature, vol. 509, no. 7498, pp. 105-109, 2014.

[8] G. Biasiotto, D. di Lorenzo, S. Archetti, and I. Zanella, "Iron and neurodegeneration: is ferritinophagy the link?," Molecular Neurobiology, vol. 53, no. 8, pp. 5542-5574, 2016. 
[9] J. D. Mancias and A. C. Kimmelman, "Mechanisms of selective autophagy in normal physiology and cancer," Journal of Molecular Biology, vol. 428, no. 9, pp. 1659-1680, 2016.

[10] T. Asano, M. Komatsu, Y. Yamaguchi-Iwai, F. Ishikawa, N. Mizushima, and K. Iwai, "Distinct mechanisms of ferritin delivery to lysosomes in iron-depleted and iron-replete cells," Molecular and Cellular Biology, vol. 31, no. 10, pp. 20402052, 2011.

[11] I. de Domenico, D. M. Ward, and J. Kaplan, "Specific iron chelators determine the route of ferritin degradation," Blood, vol. 114, no. 20, pp. 4546-4551, 2009.

[12] C. Ott, J. König, A. Höhn, T. Jung, and T. Grune, "Reduced autophagy leads to an impaired ferritin turnover in senescent fibroblasts," Free Radical Biology \& Medicine, vol. 101, pp. 325-333, 2016.

[13] Y. Fu, Y. Liu, J. Wang et al., "Calcium release induced by 2-pyridinecarboxaldehyde thiosemicarbazone and its copper complex contributes to tumor cell death," Oncology Reports, vol. 37, no. 3, pp. 1662-1670, 2017.

[14] T. Wang, Y. Liu, Y. Fu et al., “Antiproliferative activity of di-2pyridylhydrazone dithiocarbamate acetate partly involved in p53 mediated apoptosis and autophagy," International Journal of Oncology, vol. 51, no. 6, pp. 1909-1919, 2017.

[15] X. Yan, Y. Yu, P. Ji, H. He, and C. Qiao, "Antitumor activity of endoperoxide-iron chelator conjugates-design, synthesis and biological evaluation," European Journal of Medicinal Chemistry, vol. 102, pp. 180-187, 2015.

[16] M. Whitnall, J. Howard, P. Ponka, and D. R. Richardson, "A class of iron chelators with a wide spectrum of potent antitumor activity that overcomes resistance to chemotherapeutics," Proceedings of the National Academy of Sciences of the United States of America, vol. 103, no. 40, pp. 14901-14906, 2006.

[17] M. Fryknäs, X. Zhang, U. Bremberg et al., "Iron chelators target both proliferating and quiescent cancer cells," Scientific Reports, vol. 6, no. 1, article 38343, 2016.

[18] H. Glickstein, R. B. El, M. Shvartsman, and Z. I. Cabantchik, "Intracellular labile iron pools as direct targets of iron chelators: a fluorescence study of chelator action in living cells," Blood, vol. 106, no. 9, pp. 3242-3250, 2005.

[19] C. Li, Y. Liu, Y. Fu, T. Huang, L. Kang, and C. Li, “The antiproliferative activity of di-2-pyridylketone dithiocarbamate is partly attributed to catalase inhibition: detailing the interaction by spectroscopic methods," Molecular BioSystems, vol. 13, no. 9, pp. 1817-1826, 2017.

[20] F. Bou-Abdallah, J. McNally, X. X. Liu, and A. Melman, "Oxygen catalyzed mobilization of iron from ferritin by iron(III) chelate ligands," Chemical Communications, vol. 47, no. 2, pp. 731-733, 2011.

[21] T. Huang, C. Li, X. Sun et al., "The antitumor mechanism of di-2-pyridylketone 2-pyridine carboxylic acid hydrazone and its copper complex in ROS generation and topoisomerase inhibition, and hydrazone involvement in oxygen-catalytic iron mobilization," International Journal of Oncology, vol. 47, no. 5, pp. 1854-1862, 2015.

[22] J. Lin, Z. Huang, H. Wu et al., "Inhibition of autophagy enhances the anticancer activity of silver nanoparticles," Autophagy, vol. 10, no. 11, pp. 2006-2020, 2014.

[23] E. Donohue, A. Thomas, N. Maurer et al., "The autophagy inhibitor verteporfin moderately enhances the antitumor activity of gemcitabine in a pancreatic ductal adenocarcinoma model," Journal of Cancer, vol. 4, no. 7, pp. 585-596, 2013.
[24] D. B. Munafó and M. I. Colombo, "A novel assay to study autophagy: regulation of autophagosome vacuole size by amino acid deprivation," Journal of Cell Science, vol. 114, pp. 3619-3629, 2001.

[25] O. Kakhlon and Z. I. Cabantchik, "The labile iron pool: characterization, measurement, and participation in cellular processes(1)," Free Radical Biology \& Medicine, vol. 33, no. 8, pp. 1037-1046, 2002.

[26] X.-P. Dong, X. Cheng, E. Mills et al., "The type IV mucolipidosis-associated protein TRPML1 is an endolysosomal iron release channel," Nature, vol. 455, no. 7215, pp. 992-996, 2008.

[27] W. Wang, X. Zhang, Q. Gao, and H. Xu, "TRPML1: an ion channel in the lysosome," Handbook of Experimental Pharmacology, vol. 222, pp. 631-645, 2014.

[28] X. Zhang, X. Cheng, L. Yu et al., "MCOLN1 is a ROS sensor in lysosomes that regulates autophagy," Nature Communications, vol. 7, article 12109, 2016.

[29] A. Terman and T. Kurz, "Lysosomal iron, iron chelation, and cell death," Antioxidants \& Redox Signaling, vol. 18, no. 8, pp. 888-898, 2013.

[30] A. C. Gasparovic, M. Jaganjac, B. Mihaljevic, S. B. Sunjic, and N. Zarkovic, "Assays for the measurement of lipid peroxidation," Methods in Molecular Biology, vol. 965, pp. 283-296, 2013.

[31] M. U. Muckenthaler, B. Galy, and M. W. Hentze, "Systemic iron homeostasis and the iron-responsive element/ironregulatory protein (IRE/IRP) regulatory network," Annual Review of Nutrition, vol. 28, no. 1, pp. 197-213, 2008.

[32] A. La, T. Nguyen, K. Tran et al., "Mobilization of iron from ferritin: new steps and details," Metallomics, vol. 10, no. 1, pp. 154-168, 2018.

[33] M. Guo, C. Perez, Y. Wei et al., "Iron-binding properties of plant phenolics and cranberry's bio-effects," Dalton Transactions, vol. 43, no. 43, pp. 4951-4961, 2007.

[34] D. S. Kalinowski and D. R. Richardson, "Future of toxicology-iron chelators and differing modes of action and toxicity: the changing face of iron chelation therapy," Chemical Research in Toxicology, vol. 20, no. 5, pp. 715-720, 2007.

[35] V. Corcé, S. G. Gouin, S. Renaud, F. Gaboriau, and D. Deniaud, "Recent advances in cancer treatment by iron chelators," Bioorganic \& Medicinal Chemistry Letters, vol. 26, no. 2, pp. 251-256, 2016.

[36] R. J. Bergeron, J. Wiegand, N. Bharti, J. S. McManis, and S. Singh, "Desferrithiocin analogue iron chelators: iron clearing efficiency, tissue distribution, and renal toxicity," Biometals, vol. 24, no. 2, pp. 239-258, 2011.

[37] S. Sheth, "Iron chelation: an update," Current Opinion in Hematology, vol. 21, no. 3, pp. 179-185, 2014.

[38] R. J. Bergeron, J. Wiegand, N. Bharti, and J. S. McManis, "Substituent effects on desferrithiocin and desferrithiocin analogue iron-clearing and toxicity profiles," Journal of Medicinal Chemistry, vol. 55, no. 16, pp. 7090-7103, 2012.

[39] S. Omiya, S. Hikoso, Y. Imanishi et al., "Downregulation of ferritin heavy chain increases labile iron pool, oxidative stress and cell death in cardiomyocytes," Journal of Molecular and Cellular Cardiology, vol. 46, no. 1, pp. 59-66, 2009.

[40] Y. Li, M. Chen, Y. Xu et al., "Iron-mediated lysosomal membrane permeabilization in ethanol-induced hepatic oxidative damage and apoptosis: protective effects of 
quercetin," Oxidative Medicine and Cellular Longevity, vol. 2016, Article ID 4147610, 15 pages, 2016.

[41] N. Bresgen and P. Eckl, "Oxidative stress and the homeodynamics of iron metabolism," Biomolecules, vol. 5, no. 2, pp. 808-847, 2015.

[42] A. Hamacher-Brady, H. A. Stein, S. Turschner et al., "Artesunate activates mitochondrial apoptosis in breast cancer cells via iron-catalyzed lysosomal reactive oxygen species production," Journal of Biological Chemistry, vol. 286, no. 8, pp. 6587-6601, 2011.

[43] X. P. Dong, X. Cheng, E. Mills et al., "The type IV mucolipidosis-associated protein TRPML1 is an endolysosomal iron release channel," Nature, vol. 455, no. 7215, pp. 992-996, 2008.

[44] D. A. Zeevi, A. Frumkin, and G. Bach, "TRPML and lysosomal function," Biochimica et Biophysica Acta (BBA) - Molecular Basis of Disease, vol. 1772, no. 8, pp. 851-858, 2007.

[45] S. Kwiecien, K. Jasnos, M. Magierowski et al., "Lipid peroxidation, reactive oxygen species and antioxidative factors in the pathogenesis of gastric mucosal lesions and mechanism of protection against oxidative stress - induced gastric injury," Journal of Physiology and Pharmacology, vol. 65, no. 5, pp. 613-622, 2014.

[46] H. Zhong and H. Yin, "Role of lipid peroxidation derived 4-hydroxynonenal (4-HNE) in cancer: focusing on mitochondria," Redox Biology, vol. 4, pp. 193-199, 2015.

[47] X. Guo, Y. Fu, Z. Wang et al., "Di-2-pyridylhydrazone dithiocarbamate butyric acid ester exerted its proliferative inhibition against gastric cell via ROS-mediated apoptosis and autophagy," Oxidative Medicine and Cellular Longevity, vol. 2018, Article ID 4950705, 11 pages, 2018.

[48] Z. Qi, M. Wu, Y. Fu et al., "Palmitic acid curcumin ester facilitates protection of neuroblastoma against oligomeric A 340 insult," Cellular Physiology and Biochemistry, vol. 44, no. 2, pp. 618-633, 2017. 


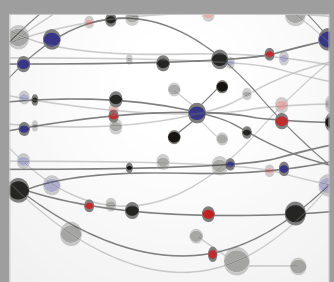

The Scientific World Journal
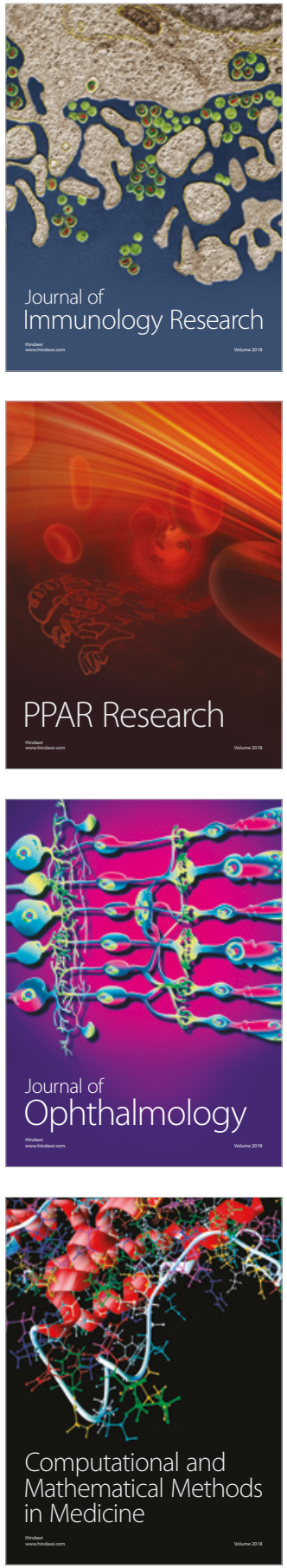

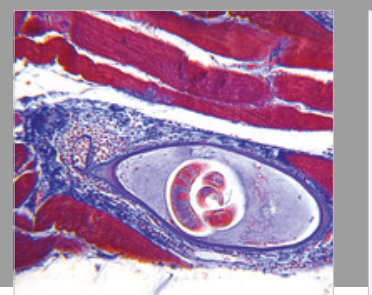

Gastroenterology Research and Practice

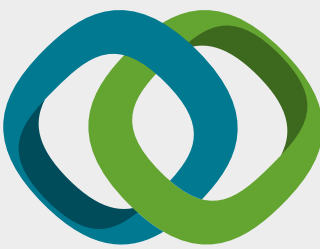

\section{Hindawi}

Submit your manuscripts at

www.hindawi.com
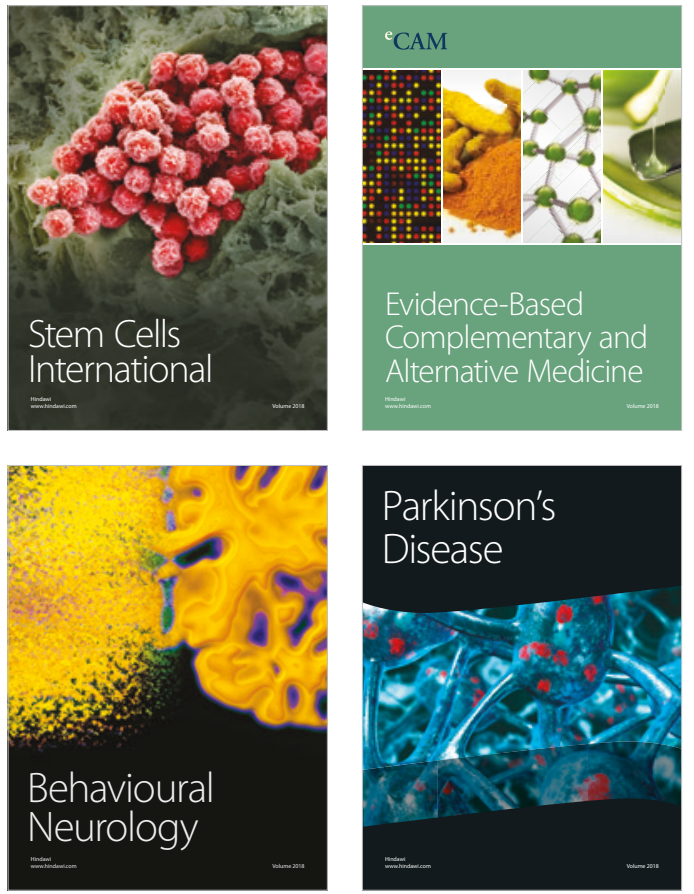

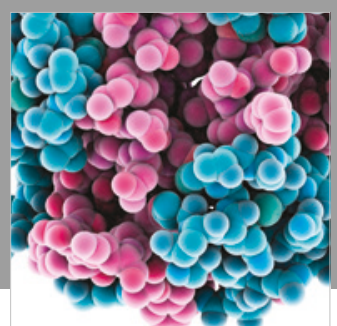

ournal of

Diabetes Research

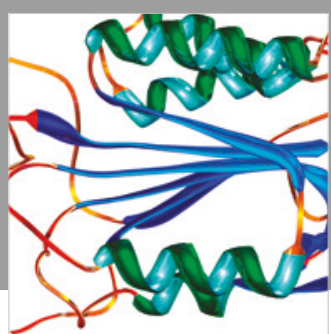

Disease Markers
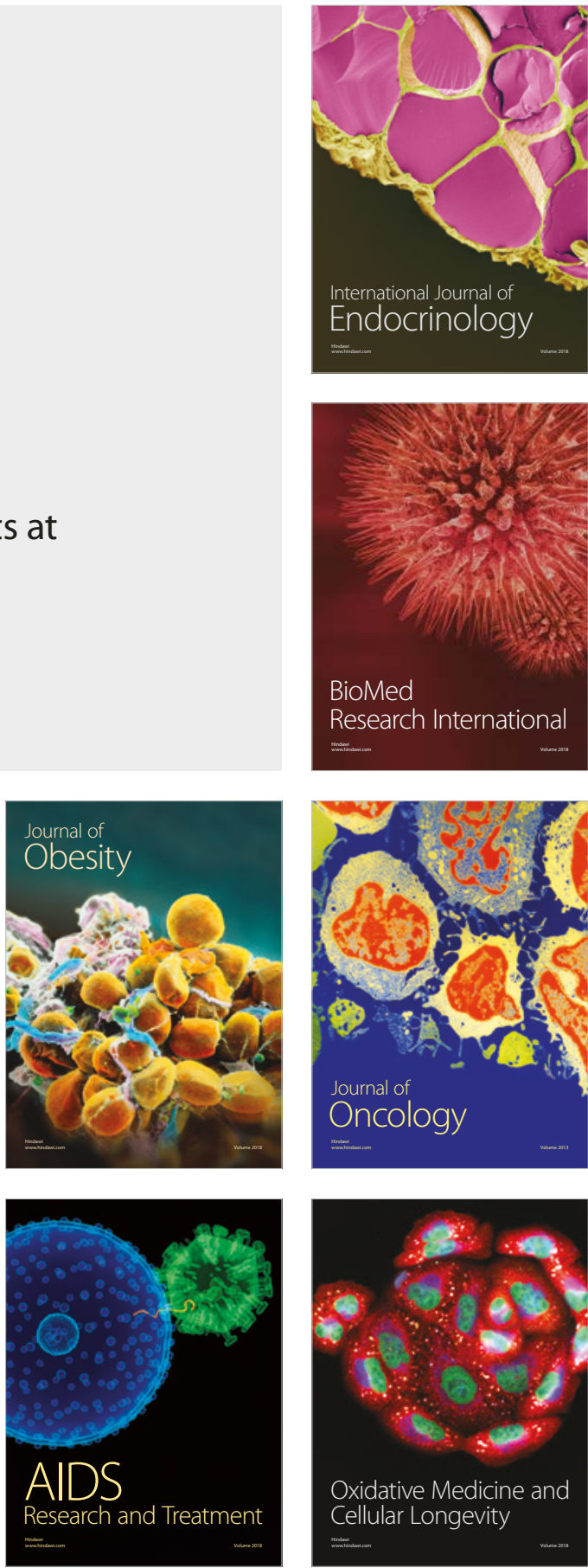